\title{
Role of Non-Steroidal Anti-Inflammatory Drugs in Exacerbations of Inflammatory Bowel Disease
}

\author{
Millie D. Long, MD, MPH ${ }^{1}$, Michael D. Kappelman, MD, MPH ${ }^{1}$, Christopher F. Martin, MSPH ${ }^{1}$, \\ Wenli Chen, MA, MS ${ }^{1}$, Kristen Anton, MS ${ }^{1,2}$, and Robert S. Sandler, MD, MPH ${ }^{1}$ \\ ${ }^{1}$ University of North Carolina at Chapel Hill, Department of Medicine, Division of Gastroenterology \\ and Hepatology
}

${ }^{2}$ Geisel School of Medicine at Dartmouth

\section{Abstract}

GOALS-To determine the role of NSAIDs in activation of IBD.

BACKGROUND—Non-steroidal anti-inflammatory drugs (NSAIDs) may activate inflammatory pathways in inflammatory bowel disease (IBD).

STUDY-Crohn's and Colitis Foundation of American (CCFA) Partners is an ongoing cohort study of patients living with IBD. All data are self-reported via the internet. We identified a subcohort of participants whose disease activity, based on short Crohn's Disease Activity Index (sCDAI) and simple clinical colitis activity index (SCCAI), indicated remission. Pattern of use of NSAIDs was measured at baseline, and disease activity assessment was performed 6 months later. We used multivariate binomial regression to determine effects of NSAIDs on disease activity.

RESULTS-A total of 791 individuals in remission had baseline and follow data available for analysis. Of these, 247 Crohn's disease (CD) patients (43.2\%) and 89 ulcerative colitis (UC) patients $(40.6 \%)$ reported NSAID use. CD patients with NSAID use $\geq 5$ times/monthly had greater risk of active disease at follow-up (23\% v. $15 \%, \mathrm{p}=0.04)$; (adjusted risk ratio (RR) $1.65 ; 95 \%$ confidence interval (CI) $1.12-2.44)$. No effect was observed in patients with UC (22\% vs $21 \%$,

Corresponding Author: Millie D. Long MD, MPH, Campus Box 7080, University of North Carolina at Chapel Hill, Chapel Hill, NC 27599-7080, millie_long@med.unc.edu, Phone: 919-843-5795, Fax: 919-966-6842.

Conference presentation: This work was presented as an oral plenary presentation at the American College of Gastroenterology meeting, October 2013.

Author contribution: All authors have made substantial contributions to this work. MDL participated in all of the following: (1) the conception and design of the study, acquisition of data, analysis and interpretation of data, (2) drafting the article or revising it critically for important intellectual content, (3) final approval of the version to be submitted. MDK participated in 1) the conception and design of the study, (2) final approval of the version to be submitted. CFM participated in 1) the conception and design of the study, acquisition of data, analysis and 2) final approval of the version to be submitted. WC participated in 1) acquisition of data, 2) final approval of the version to be submitted. KA participated in 1) acquisition of data, 2) final approval of the version to be submitted. RSS participated in 1) the conception and design of the study, (2) final approval of the version to be submitted.

Financial disclosures:

1. Millie Long MD MPH served as an advisory board member for Abbvie, NPS and Janssen. She has consulted for Salix.

2. Michael D. Kappelman has served as a consultant to GlaxoSmithKline.

The writing of this paper was funded in part by a grant from the CCFA for development and management of CCFA Partners Internet Cohort. 
$\mathrm{p}=0.98$; adjusted RR 1.25; 95\% CI, 0.81-1.92). Acetaminophen use was associated with active disease at follow-up in CD (adjusted RR 1.72, 95\% CI 1.11-2.68).

CONCLUSIONS-Regular ( $\geq 5$ times/monthly) NSAID and acetaminophen use were associated with active CD, but not UC. Less frequent NSAID use was not associated with active CD or UC. These findings indicate that regular NSAID use may increase CD activity, or that NSAID use may be a marker of a less robust remission; thus reflecting subclinical disease activity.

\section{Introduction}

Inflammatory bowel diseases (IBD), including Crohn's disease (CD) and ulcerative colitis (UC) are chronic relapsing inflammatory disorders of the gastrointestinal tract of unknown etiology. These diseases are thought to arise via a combination of genetic, environmental and microbial (microbiota) effects. ${ }^{1,2}$ Unfortunately, causative agents of relapses of inflammation in these disorders are not well characterized. Etiologies of disease relapse are a high priority for research. ${ }^{3}$

Non-steroidal anti-inflammatory drugs (NSAIDs) are a class of pain medications that have been associated with increased risk of IBD development. ${ }^{4}$ NSAIDs are also known to cause enteropathy and gastropathy, which could contribute to or trigger disease exacerbation. The proposed mechanisms associated with these effects include decreased production of prostaglandins (through both cyclooxygenase (COX) 1 and COX-2 pathways) and topical actions via surface membrane phospholipid interaction and effects on mitochondrial activity. ${ }^{5,6}$ It is possible that these medications play a role in activation of inflammatory pathways in IBD through similar mechanisms.

Prior data on NSAID use and relapse of IBD are conflicting. Data in the form of caseseries, ${ }^{7,8}$ a case control study with hospitalization for IBD as the outcome, ${ }^{9}$ and one small $\operatorname{trial}^{6}$ demonstrated a detrimental effect of NSAID use in individuals with IBD. The trial demonstrated a 17-28\% relapse rate within 9 days of ingestion. Other studies, including cross-sectional $^{10}$ and cohort studies ${ }^{11,12}$ found no effect of NSAIDs on disease activity.

Patients with IBD may have a number of extra-intestinal conditions including painful axial and peripheral arthopathy. Even though the literature on IBD exacerbation from NSAIDs is limited, patients are often instructed by their physicians to avoid these inexpensive and possibly helpful medications. In order to learn more about the association between NSAIDS and IBD exacerbation we examined data from a large IBD cohort study. We aimed to determine whether NSAID use increases the risk of increased disease activity in a population of patients with IBD in remission.

\section{Materials and Methods}

Crohn's and Colitis Foundation of American (CCFA) Partners is an ongoing cohort study of patients living with IBD. All data are self-reported via the internet. ${ }^{13}$ Following a baseline survey, participants complete online surveys every 6 months. Data on demographics, disease type and characteristics, medications, disease activity (via the short Crohn's disease activity index (sCDAI) for CD and the simple clinical colitis activity index (SCCAI) for UC as well 
as the Manitoba Index) and other patient reported outcomes are collected at each interval. We performed a cohort study nested within CCFA Partners. For inclusion, individuals had to 1) have IBD, 2) be in remission at baseline (defined as SCDAI $<150$ or SCCAI ㅅ) 3) provide complete NSAID and non-NSAID pain medication histories at baseline and 4) not change diagnoses (CD or UC) during the study. A similar design has been used in another study within this cohort of factors associated with relapse of IBD. ${ }^{14}$

The main exposure was NSAID use. Data were collected on current use of NSAIDs and average number of doses per month. Similar data were collected for non-NSAID pain medications (acetaminophen). Exposure definitions were determined a priori, and included categories of any/none and $\geq 5$ times/monthly versus $<5$ times/monthly. The cutoff of 5 times monthly was felt to represent "regular" use, due to an average of greater than once weekly use. The main outcome of interest was active disease at 6 months of follow-up, defined by sCDAI $\geq 150$ and SCCAI $>2$. Alternate outcomes were investigated in sensitivity analyses, including sCDAI >250 and SCCAI $>4$ and the Manitoba index for IBD.

Bivariate analyses were used to calculate associations between exposures and outcome. Binomial regression models were used to control for potential confounders and to determine the independent effects of NSAIDs on active disease at follow-up. All statistical analyses were performed using Stata version 11.0 (College Station, TX, USA). For all analyses, pvalues were two-sided, and a p-value of 0.05 or less was considered statistically significant. The study protocol was approved by the Institutional Review Board at the University of North Carolina.

\section{Results}

A total of 1617 individuals with IBD met inclusion criteria for the study. Of these, 791 (49\%) completed a follow-up survey. When comparing the 826 on whom no follow-up information was available to those who did complete follow-up, there was no substantial difference between NSAID use or other baseline characteristics between the groups. The entire population met criteria for remission at baseline, with a median cCDAI of 93 (cut off for remission <150) for CD patients and mean SCCAI of 1.1 (cut off for remission of $\mathfrak{2}$ ) for UC patients. There was no significant difference in SCDAI or SCCAI at baseline when stratified by any NSAID use. A total of 572 individuals (72.3\%) had CD and 219 (27.7\%) had UC. Of these, 336 (42.5\%) reported any use of NSAIDs at baseline, with similar rates of any NSAID usage for both CD and UC patients (43.2\% and 40.7\%, respectively). Overall, NSAID uses/month ranged from 1-180 times per month. The median NSAID uses/month was 4.3 (IQR 2-13). The characteristics of the population by any NSAID use are shown in table 1 . In general, the 2 groups were similar without specific factors associated with NSAID exposure.

When evaluating categories of pain medication use, very few (3.8\%) used prescription NSAIDs (selective COX-2 inhibitors, diclofenac, indomethacin, meloxicam, etc), 19.3\% reported $\geq 5$ uses/month ("regular" use) of NSAIDs, and nearly $2 / 3$ reported acetaminophen use (64.6\%) (figure 1). On bivariate analysis, for the IBD population overall, there was no significant difference between any versus no NSAID use or $\geq 5$ times monthly versus $<5$ 
times monthly NSAID use and active disease at follow up (19\% v. $17 \%, \mathrm{p}=0.54$ and $23 \% \mathrm{v}$. $17 \%, \mathrm{p}=0.09$, respectively). However, when stratified by $\mathrm{CD}$ or UC, there was a significant difference in disease activity at follow-up among those with CD reporting $\geq 5$ uses/month of NSAIDS, (23\% v. $15 \%, \mathrm{p}=0.04)$. Interestingly, there was also an increased risk of active disease at follow-up for CD patients reporting acetaminophen use at baseline (20\% v. $11 \%$, $\mathrm{p}=0.01$ ). For patients with UC, there was no difference in active disease at follow up for any NSAID use versus none ( $24 \%$ vs $20 \%, \mathrm{p}=0.52$ ) or for $\geq 5$ times/monthly NSAID use versus $<5$ times/monthly ( $22 \%$ vs $21 \%$, $\mathrm{p}=0.98$ ) (figure 2 ). Unadjusted risk ratios for active disease at follow-up, stratified by disease type, are shown in table 2 .

In binomial regression models controlling for IBD medication use, smoking, sex, age and other NSAID or acetaminophen use, individuals with CD with $\geq 5$ uses/month of NSAIDS had significantly increased risk of active disease at follow-up (adjusted risk ratio (RR) of 1.65; 95\% confidence interval (CI) 1.12-2.44.) Acetaminophen use at baseline among individuals with $\mathrm{CD}$ was also independently associated with active disease at follow-up (adjusted RR 1.72; 95\% CI 1.11-2.68). No independent association was found between regular use ( $\geq 5$ uses/month) of NSAIDs at baseline and active disease at follow up for UC (adjusted RR 1.25; 95\% CI 0.82-1.92). Acetaminophen use at baseline also was not associated with active disease at follow up for UC (adjusted RR 1.09; 95\% CI 0.75-1.57).

In sensitivity analyses evaluating alternate outcomes, similar trends were seen in the IBD population as a whole using the Manitoba index as the outcome (increased activity defined as sometimes, often or constant activity at follow-up). When we evaluated only the CD population for this outcome, $23.2 \%$ with 25 uses NSAIDs/month had active disease at follow up, compared to $18.9 \%$ with $<5$ uses NSAIDs/month ( $\mathrm{p}=0.23$ ). Using the outcome of sCDAI $>250$ for active disease, similar trends were again seen with more active disease among those with "regular" use, although none reached statistical significance. Using this definition requiring a higher level of active disease in $\mathrm{CD}, 6.9 \%$ of those with NSAID use $\geq 5$ times monthly had active disease, as compared to $4.0 \%$ of those with NSAID use $<5$ times/monthly $(\mathrm{p}=0.17)$.

\section{Discussion}

In this study of NSAID and acetaminophen use at baseline and active disease in patients with IBD, we observed several important findings. First, use of NSAIDs among patients with IBD is common, despite the fact that many gastroenterologists caution against NSAID use in IBD patients. We also found that low doses of NSAIDS in patients with CD, or any NSAID use in those with UC, do not appear to impact disease activity. However, higher or "regular" NSAID doses ( $\geq 5$ times monthly) are associated with active disease at follow-up among patients with $\mathrm{CD}$ in our study.

The prevalence of NSAID use in this internet-based cohort of predominantly individuals in the United States was similar to that previously reported in a population-based cohort from Canada. ${ }^{12}$ The literature has been conflicting as to the effects of NSAIDs, but this may be partially explained by a possible dose-response effect with NSAIDs and small numbers in prior studies, particularly for subgroup analyses. Because of the large size of our study and 
our detailed information about NSAIDs we were able to identify differing effects with a higher dose (using a threshold of $\geq 5$ times/monthly). Study designs have also differed, with varying definitions of active disease or using proxy outcomes, such as hospitalization. The timing of NSAID use is also important, in that measurement of NSAID exposure at the time of a flare could be a consequence of the flare (due to abdominal or joint pain from inflammation) rather than a cause of the flare. For example, in a case-control study comparing those with UC admitted to the hospital, as compared to community controls, more individuals admitted to the hospital had recent or current NSAID use (OR 1.7-1.9). ${ }^{9}$ However, NSAID use was measured in close proximity to the flare. In our study, we established use patterns at baseline while the individual was in remission, which allows us to separate NSAID use patterns from management of symptoms of a flare. Felder et at previously reported an association between NSAID use and IBD activity. ${ }^{15}$ Bernstein et al ${ }^{12}$ demonstrated no increased risk of flare associated with NSAID use in a prospective IBD cohort in Manitoba. There was no evidence of a dose-response effect of NSAIDs. However, the population was not stratified by UC and CD to determine if there were disease-specific differences in flares by NSAID use. Bonner et al ${ }^{11}$ found no increase in disease activity associated with low dose NSAID use in CD or UC populations, similar to our findings. High dose NSAIDs were associated with higher numerical disease activity scores in CD patients, particularly for those with colonic involvement. ${ }^{11}$ These data and our data support a possible dose-response effect in those with $\mathrm{CD}$.

There are several strengths of this study. First, the prospective design minimized the possibility of reverse causation, i.e. that NSAID exposure was impacted by the presence of active disease. This is important, as flares of disease can be accompanied by abdominal and joint pain, for which NSAIDs may be used. Additionally, the large sample size allowed us to separately analyze associations in patients with $\mathrm{CD}$ and $\mathrm{UC}$, as is appropriate for biologically distinct conditions. Finally, we used validated indices for self-reported measurement of disease activity. ${ }^{16,17}$

There are also limitations to this study. First, our cohort is a self-reported cohort of individuals with IBD, and hence disease type is subject to misclassification. However, a recent validation study within CCFA Partners that found $>95 \%$ accuracy of self-reported IBD diagnosis when compared to physician report. ${ }^{18}$ Also, there was substantial loss to follow-up within the cohort, which could have biased our results if this were differential. However, baseline characteristics and exposure status between those who did and who did not follow-up were not substantially different, suggesting that loss to follow-up was likely non-differential. We also did not have objective measures of inflammation, and factors such as endoscopic or laboratory evidence of inflammation were not available to confirm disease activity. Additionally, data were not available as to the indication for NSAID or acetaminophen use (headache, menorrhagia, abdominal pain, joint pain, etc) and NSAID exposure data were by self-report. We intentionally evaluated NSAID exposure patterns remote from a flare, in a population in remission, so as not to confound the association. Higher NSAID use immediately prior to a flare may be related to arthralgias or other pain directly related to the flare itself. However, it is possible that use patterns measured at baseline may not be consistent throughout the exposure window. A prior study by Bonner et al demonstrated an association with high dose NSAIDS and increased disease activity in 
patients with colonic involvement of CD. Unfortunately, we were not able to stratify our analyses by disease location. Finally, this was a relatively short-term study with only 6 months of follow-up. However, this time estimate is likely reasonable to study a short acting exposure such as pain medication use.

There are several implications to this study. First, the requirement for any pain medication while in remission may be a marker of occult disease. This occult disease may in fact be the risk factor for relapse, rather than the NSAID or acetaminophen. This is important to clinical care, as physicians should be suspicious that patients who appear to be in clinical remission yet require NSAIDS may have ongoing inflammation. For those with the requirement for regular use of these medications, further investigation may be warranted to determine if inflammation is present. It is also possible that a mechanism common to NSAIDS and acetaminophen may play a role in inflammation development in IBD. Importantly, we now also know that infrequent NSAID use does not increase disease activity for those in clinical remission with $\mathrm{UC}$ or $\mathrm{CD}$, and can potentially be used intermittently without adverse consequences. Certainly, further prospective studies with objective inflammatory outcomes and mechanistic studies are needed to assess the risks of these pain medications during remission. Data gleaned from this study will enable providers to better counsel IBD patients on intermittent use of analgesics.

\section{Acknowledgments}

Grant support: This work was supported by a grant from the Crohn's and Colitis Foundation of America, NIH P30 DK34987 and NIH 1K08DK088957-01.

\section{References}

1. Ananthakrishnan AN. Environmental triggers for inflammatory bowel disease. Curr Gastroenterol Rep. 2013; 15:302. [PubMed: 23250702]

2. Ponder A, Long MD. A clinical review of recent findings in the epidemiology of inflammatory bowel disease. Clin Epidemiol. 2013; 5:237-47. [PubMed: 23922506]

3. Denson LA, Long MD, McGovern DP, et al. Challenges in IBD research: update on progress and prioritization of the CCFA's research agenda. Inflamm Bowel Dis. 2013; 19:677-82. [PubMed: 23448796]

4. Ananthakrishnan AN, Higuchi LM, Huang ES, et al. Aspirin, nonsteroidal anti-inflammatory drug use, and risk for Crohn disease and ulcerative colitis: a cohort study. Ann Intern Med. 2012; 156:350-9. [PubMed: 22393130]

5. Meyer AM, Ramzan NN, Heigh RI, Leighton JA. Relapse of inflammatory bowel disease associated with use of nonsteroidal anti-inflammatory drugs. Dig Dis Sci. 2006; 51:168-72. [PubMed: 16416231]

6. Takeuchi K, Smale S, Premchand P, et al. Prevalence and mechanism of nonsteroidal antiinflammatory drug-induced clinical relapse in patients with inflammatory bowel disease. Clin Gastroenterol Hepatol. 2006; 4:196-202. [PubMed: 16469680]

7. Rampton DS, Sladen GE. Relapse of ulcerative proctocolitis during treatment with non-steroidal anti-inflammatory drugs. Postgrad Med J. 1981; 57:297-9. [PubMed: 6117846]

8. Kaufmann HJ, Taubin HL. Nonsteroidal anti-inflammatory drugs activate quiescent inflammatory bowel disease. Ann Intern Med. 1987; 107:513-6. [PubMed: 3498419]

9. Evans JM, McMahon AD, Murray FE, McDevitt DG, MacDonald TM. Non-steroidal antiinflammatory drugs are associated with emergency admission to hospital for colitis due to inflammatory bowel disease. Gut. 1997; 40:619-22. [PubMed: 9203940] 
10. Bonner GF, Walczak M, Kitchen L, Bayona M. Tolerance of nonsteroidal antiinflammatory drugs in patients with inflammatory bowel disease. Am J Gastroenterol. 2000; 95:1946-8. [PubMed: 10950040]

11. Bonner GF, Fakhri A, Vennamaneni SR. A long-term cohort study of nonsteroidal antiinflammatory drug use and disease activity in outpatients with inflammatory bowel disease. Inflamm Bowel Dis. 2004; 10:751-7. [PubMed: 15626893]

12. Bernstein CN, Singh S, Graff LA, Walker JR, Miller N, Cheang M. A prospective populationbased study of triggers of symptomatic flares in IBD. Am J Gastroenterol. 2010; 105:1994-2002. [PubMed: 20372115]

13. Long MD, Kappelman MD, Martin CF, et al. Development of an internet-based cohort of patients with inflammatory bowel diseases (CCFA Partners): methodology and initial results. Inflamm Bowel Dis. 2012; 18:2099-106. [PubMed: 22287300]

14. Ananthakrishnan AN, Long MD, Martin CF, Sandler RS, Kappelman MD. Sleep disturbance and risk of active disease in patients with Crohn's disease and ulcerative colitis. Clin Gastroenterol Hepatol. 2013; 11:965-71. [PubMed: 23376797]

15. Felder JB, Korelitz BI, Rajapakse R, Schwarz S, Horatagis AP, Gleim G. Effects of nonsteroidal antiinflammatory drugs on inflammatory bowel disease: a case-control study. Am J Gastroenterol. 2000; 95:1949-54. [PubMed: 10950041]

16. Jowett SL, Seal CJ, Phillips E, Gregory W, Barton JR, Welfare MR. Defining relapse of ulcerative colitis using a symptom-based activity index. Scand J Gastroenterol. 2003; 38:164-71. [PubMed: 12678333]

17. Thia K, Faubion WA Jr, Loftus EV Jr, Persson T, Persson A, Sandborn WJ. Short CDAI: development and validation of a shortened and simplified Crohn's disease activity index. Inflamm Bowel Dis. 2011; 17:105-11. [PubMed: 20629100]

18. Randell RL, Cook S, Wrennall CE, et al. 982 Validation of an Internet-Based Cohort of Patients With Inflammatory Bowel Diseases (CCFA Partners). Gastroenterology. 2013; 144:S-180. 


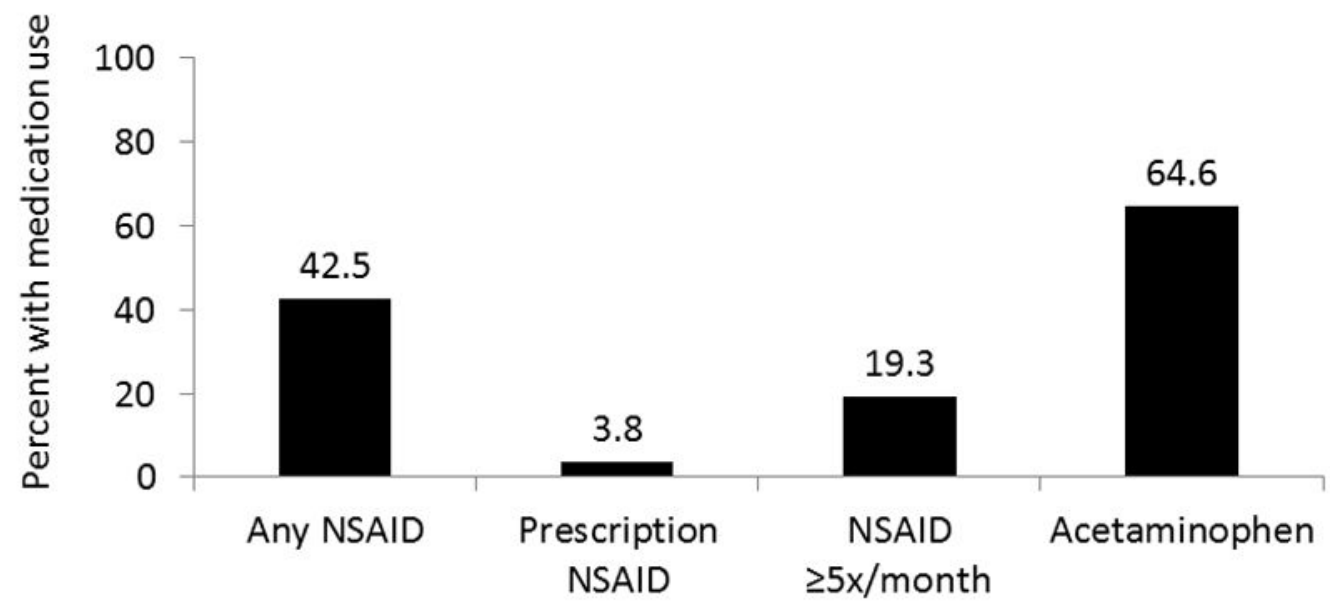

Figure 1.

Categories of pain medication use in cohort at baseline 


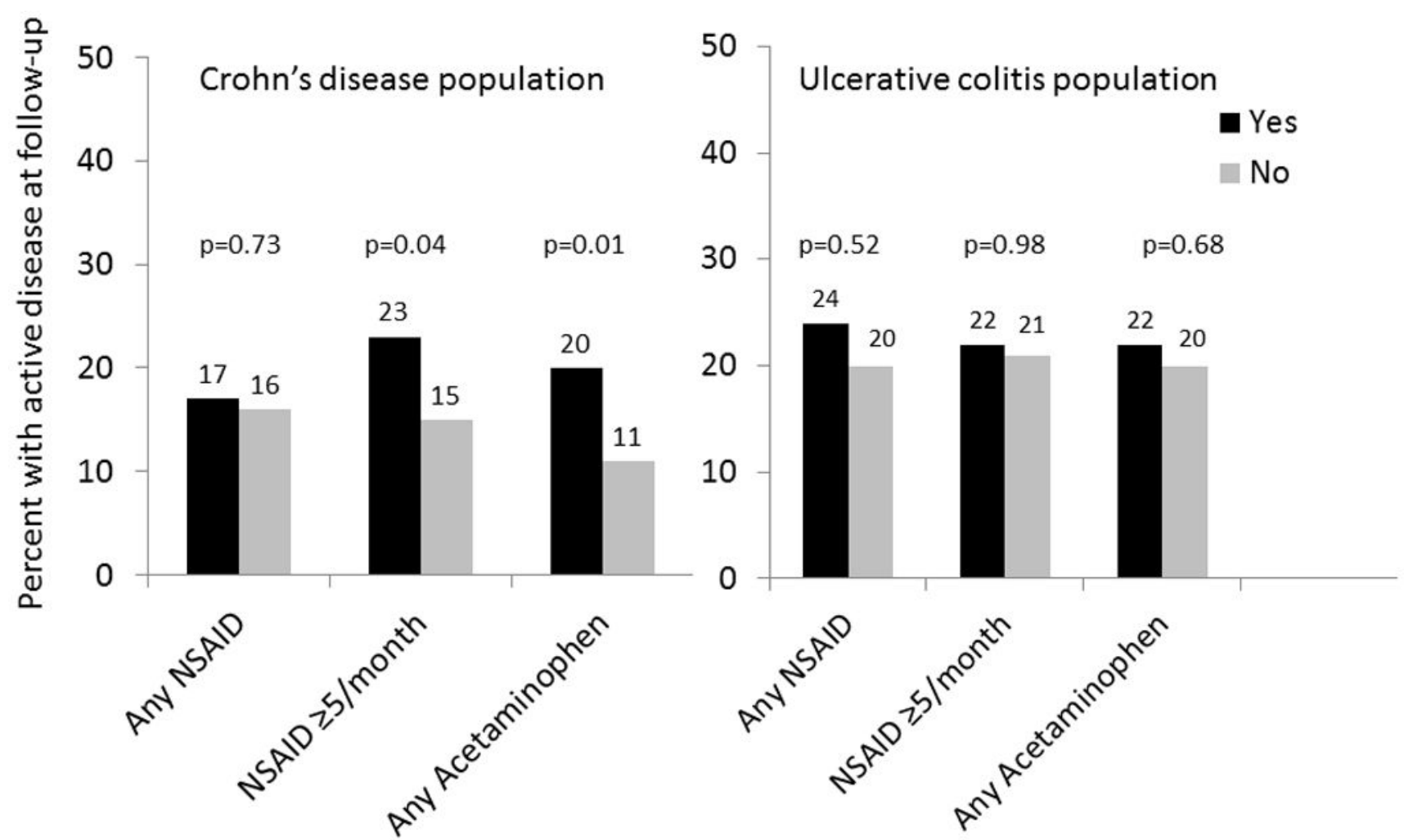

Figure 2.

Categories of pain medication use at baseline and active disease at follow-up, stratified by Crohn's disease or ulcerative colitis 


\section{Table 1}

Characteristics of the population in remission by non-steroidal anti-inflammatory drug (NSAID) use at baseline $(n=791)$

\begin{tabular}{|c|c|c|}
\hline Characteristic & $\begin{array}{c}\text { Any NSAID use }(\mathrm{n}=336) \\
\text { Mean (SD), median (IQR) or \% }\end{array}$ & $\begin{array}{c}\text { No NSAID use }(\mathrm{n}=455) \\
\text { Mean (SD), median }(\mathrm{IQR}) \text { or \% }\end{array}$ \\
\hline Age & $43.8(14.8)$ & $44.8(15.4)$ \\
\hline \multicolumn{3}{|l|}{ IBD Type } \\
\hline $\mathrm{CD}$ & 73.5 & 71.4 \\
\hline $\mathrm{UC}$ & 36.5 & 28.6 \\
\hline Gender ( $\%$ female) & 71.1 & 67.3 \\
\hline Disease duration (years) & $11(6-22)$ & $10(4-20)$ \\
\hline Smoking (\% yes) & 26.5 & 31.7 \\
\hline \multicolumn{3}{|l|}{ Medication use } \\
\hline Steroids (\% yes) ${ }^{*}$ & 10.1 & 11.4 \\
\hline Immunomodulator ${ }^{* *}$ & 24.4 & 25.3 \\
\hline Anti-TNF ${ }^{\wedge}$ & 28.8 & 36.7 \\
\hline $5-\mathrm{ASA}^{\%}$ & 53.0 & 46.8 \\
\hline \multicolumn{3}{|c|}{ * including rectal preparations and budesonide } \\
\hline \multicolumn{3}{|c|}{ ** Thiopurine: azathioprine or mercaptopurine } \\
\hline \multicolumn{3}{|c|}{ ^ anti-TNF: anti- tumor necrosis factor alpha agent } \\
\hline
\end{tabular}


Table 2

Pain medication use at baseline and risk of symptoms of active inflammatory bowel disease at follow up

\begin{tabular}{lcc}
\hline Medication & $\begin{array}{c}\text { Risk of active Crohn's disease at follow up (n=572) } \\
\text { RR (95\% CI) }\end{array}$ & $\begin{array}{c}\text { Risk of active ulcerative colitis at follow up (n=219) } \\
\text { RR (95\% CI) }\end{array}$ \\
\hline Any NSAID $* *$ & $1.05(0.82-1.34)$ & $1.13(0.78-1.63)$ \\
NSAID 25 x/monthly & $1.50(1.04-2.18)$ & $1.01(0.49-2.06)$ \\
Any acetaminophen & $1.21(1.07-1.38)$ & $1.06(0.82-1.35)$ \\
\hline$*$ &
\end{tabular}

